\title{
A New Method of Extrusion Simulations of Newtonian Fluids
}

\section{Yu Cao ${ }^{1, a}$, Miao Wang ${ }^{1, b}$ Qian Wang ${ }^{1, c}$ Chao $^{1} i^{1, d}$ Wenjing Yang ${ }^{1, e, *}$}

${ }^{1}$ State Key Laboratory of High Performance Computing, National University of Defense Technology, Changsha, Hunan, China

aemail: caoyu@nudt.edu.cn, bemail: mercury.miao@nudt.edu.cn, bemail: wangqian@nudt.edu.cn bemail: dirk911@nudt.edu.cn, bemail: wenjing.yang@nudt.edu.cn

Keywords: Free Surface; ALE; Extrusion flows; Newtonian fluids

\begin{abstract}
The simulation of free surface problems (e.g. waves, bubbles) are important in various fields. Arbitrary Lagrangian-Eulerian (ALE) is one the famous methods to track the position of free surface. However, this method is usually used in Finite Element Method (FEM) systems. The main difficulty to integrate it into Finite Volume Method (FVM) systems is how to address the free surface boundary condition. This paper proposed a new numerical method to track the movement of free surface which combined ALE and FVM. And it is validated in 2D extrusion flows with Newtonian fluids. Comparisons were made with previous literature and similar results were obtained, which indicated that the correctness and effectiveness of our method.
\end{abstract}

\section{Introduction}

In recent years, there was a growing interest in free surface problems (e.g. die- swell, bubble growing). Extrusion flow is one of the free surface problems. It was first observed in polymer processing [1-3]. When the viscoelastic fluids flows out of a die, the width or the radius of the extrudate part would become larger. However, later researchers found out that Newtonian fluids could also produce die-swell phenomena when the Reynolds number is small $[4,5]$. Investing the behavior of Newtonian fluid in die-swell is beneficial to having a better understanding of the property of Newtonian fluids.

In order to simulate the extrusion flow, a great number of methods were developed to track the position of free surface. Basically speaking, there are two methods to do so. One is interface capturing and the other is interface tracking. Typical interface capturing methods include Volume of fluid (VOF) method [6],Level set method [7], Marker and Cell (MAC) method [8] etc. In these methods, the computational grids were static and the interface was reconstructed by numerical methods. These methods could tackle with large deformation cases well, however, the accuracy is limited by the interfacial reconstruction algorithm. Though the precision could also be improved by finer meshes, it would require more cells and more computational resources. Interface tracking methods could precisely and directly track the position of free surface by moveable computational grids. The disadvantage of these methods is the difficulty to address mesh distortions in large deformation cases.

Arbitrary Lagrangian-Eulerian method [9] makes a good trade-off between the precision of free surface position and the capability of addressing large deformation cases. It not only inherits the advantage of tracking the exact interface like Lagrangian mesh, but also overcomes the failure caused by large deformation cases by constraining the moving direction and keeping the mesh in an Eulerian manner. Generally, ALE method is combined with FEM, while VOF method is combined with FVM. Combing ALE with FVM could not only track the free surface precisely, but also keep a good conservation of physical properties brought by FVM. Tukovic [10] proposed a method to simulate two-fluid systems, however, an additional mesh on the surface was generated which is time-consuming.

This paper aims at developing a simple method to simulate extrusion flows with Newtonian fluids. The difficulty of imposing a proper boundary condition on the surface is solved by an 
iteration process. The experiments is implemented in the software OpenFOAM [11], which is widely used in CFD simulations [12, 13]. Detailed description of the method is presented in Section Methodology. Numerical results are discussed afterwards. The conclusions are drawn at last.

\section{Methodology}

Mathematical model The governing equations for laminar, isothermal and incompressible Newtonian fluid can be written as

$$
\begin{aligned}
\rho\left(\frac{\partial u}{\partial t}+(u-w) \square \nabla u\right) & =-\nabla p+\eta \Delta u \\
\nabla \square u & =0
\end{aligned}
$$

Where $\rho$ is the fluid density, $p$ is the pressure, $\eta$ is the solvent viscosity. $u$ and $w$ represent the velocity of fluids and grids, respectively.

Geometry and initial conditions The behavior of Newtonian fluids flow out of a die is investigated in this paper. The geometry and initial conditions are briefly illustrated in Fig. 1, where $\sigma=-p \boldsymbol{I}+2 \eta \boldsymbol{D}$ is the total stress tensor. in which $\boldsymbol{D}$ is the rate of deformation tensor, which can be expressed as $\boldsymbol{D}=\left(\nabla u+(\nabla u)^{T}\right) / 2$. In this paper, the input velocity $u_{i n}$ is chosen as uniform flow. $L_{1}=L_{2}=0.08 \mathrm{~m}$ and $L=0.01 \mathrm{~m}$.

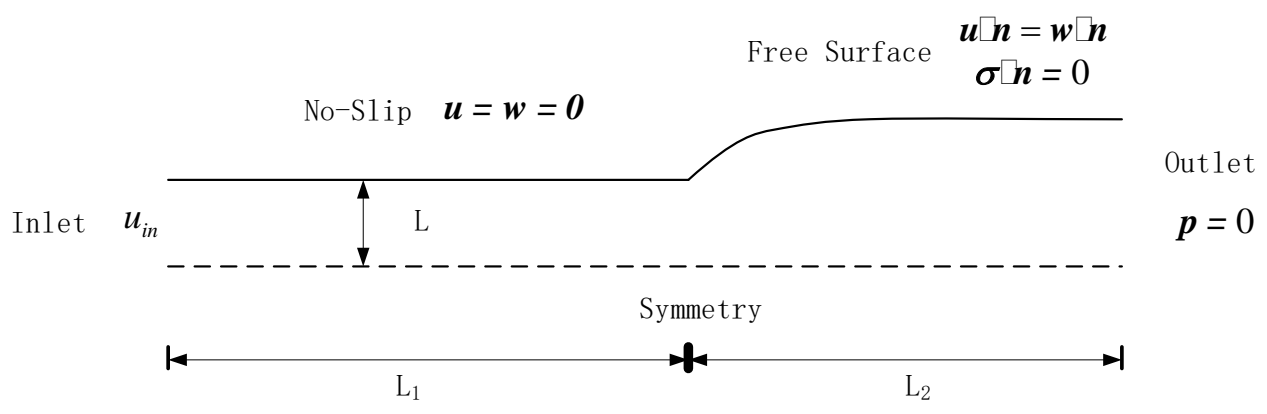

Fig.1. Die swell flow configurations

Numerical algorithm The difficulty to combine ALE methods with FVM is how to set a proper boundary condition on the free surface. The free surface boundary condition could be divided into two parts. One is the kinematic boundary condition and the other one is dynamic boundary condition. The kinematic boundary condition means the relative speed between the fluid and the surface grid normal to the interface should be equal, which can be expresses as

$$
\boldsymbol{u} \cdot \boldsymbol{n}=\boldsymbol{w} \cdot \boldsymbol{n}
$$

where $\boldsymbol{n}$ is the unit surface normal vector. This boundary condition has been implemented by Tukovic [10] in OpenFOAM. In terms of the dynamic boundary condition, the total force imposed on the surface should equal to zero, which is given by

$$
F=\boldsymbol{\sigma} \cdot \boldsymbol{n}=(-p \boldsymbol{I}+2 \eta \boldsymbol{D}) \cdot \boldsymbol{n}=0
$$

An additional mesh is generated by Tukovic [10] to calculate the velocity on the free surface which is quite complex. Therefore, we proposed a simpler method to implement the dynamic boundary condition. The boundary condition of the pressure could be obtained by multiplying $\boldsymbol{n}$ with the left side of Eq. 4, then we have

$$
p=2 \eta \boldsymbol{D}
$$

In terms of the velocity boundary on the surface, we proposed an iteration methods. The procedure can be described as follows.

Step 1: Moving the internal grids according to the movement of the free surface grids

Step 2: Based on physical properties ( $\mathrm{p}$ and $\mathrm{u}$ ) solved in the last time step, setting the proper 
pressure boundary condition on the surface according to Eq. 5, and modifying the surface velocity boundary condition by

$$
u_{f}^{n+1}=u_{f}^{n}-\alpha F
$$

where $\alpha$ is the relaxation parameter. We choose $\alpha=0.001$ in the simulations.

Step 3: Solving the latest physical properties by SIMPLE algorithm. Return to Step 2 unless $F<\varepsilon$, where $\varepsilon=10^{-3}$ is chosen in the present work.

Step 4: The surface grids is moved according to the velocity on the surface to satisfy kinematic boundary condition (details see [10]).

\section{Results and discussion}

By setting $\rho=1000 \mathrm{~kg} / \mathrm{m}^{3}, u_{i n}=0.001 \mathrm{~m} / \mathrm{s}, \eta=0.01 \mathrm{~kg} /(\mathrm{m} \cdot \mathrm{s})$, The Reynolds number, which is defined as $\operatorname{Re}=\rho u L / \eta$, equals to one. Three different meshes are used to test the mesh convergence. Details of the mesh configures and the swelling ratio $\gamma$ at $\operatorname{Re}=1$ are listed in Table 1 . Comparing with the results in [4] $(\gamma=1.19)$, our result is slightly smaller than theirs with 4800 elements. However, with the increase of mesh density, the result is approaching theirs gradually. More importantly, using mesh M1, the swelling ratio with the original algorithm proposed by Tukovic [10] is 1.152, which means that our new algorithm has made an improvement in the precision in extrusion flows. Since no significant improvement is seen between M2 and M3, and the computational time with M3 is much longer than the one with M2, the mesh M2 is used in the following simulation. A brief review of the computational mesh can be seen in Fig. 2. It can be seen that the mesh size near the wall and near the singularity is smaller than elsewhere. This is because the variation of velocity is more drastic around these areas.

Table 1 . The swelling ratio at $\mathrm{Re}=1$ with different mesh configures

\begin{tabular}{|l|l|l|l|l|}
\hline Mesh & Cells number & $(\Delta x)_{\min }\left(10^{-2}\right)$ & $(\Delta y)_{\min }\left(10^{-2}\right)$ & Swelling ratio \\
\hline M1 & 9450 & 0.067 & 0.013 & 1.158 \\
\hline M2 & 16800 & 0.05 & 0.01 & 1.163 \\
\hline M3 & 32000 & 0.025 & 0.05 & 1.170 \\
\hline
\end{tabular}

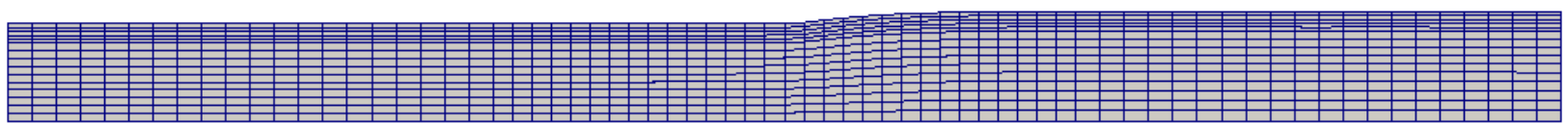

Fig.2. Computational mesh

The pressure and the $x$ component of velocity near the wall and the free surface around the singularity at $\operatorname{Re}=1$ (values in nearest cells between the range [-0.04,0.04]) are shown in Fig. 3 . It can be seen that both the pressure and the velocity would suffer a sudden change when the fluid flows out of the die exit. The surface pressure would decline to zero after the peak. In terms of the $\mathrm{x}$ component of the velocity, it would increase immediately, and reach a plateau at last. It means the flow has become a stable plug flow at present. 


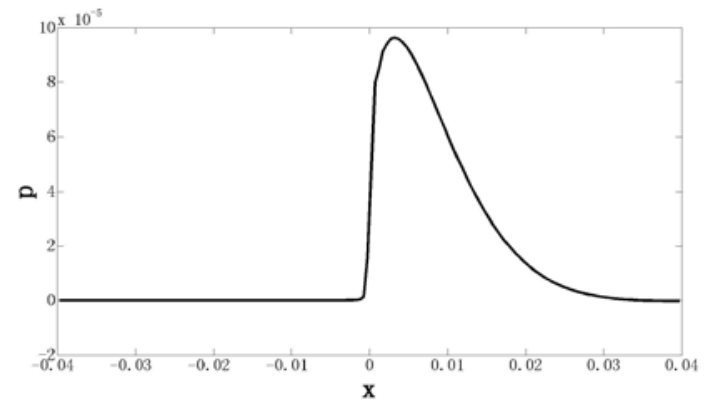

(a) $\mathrm{p}$

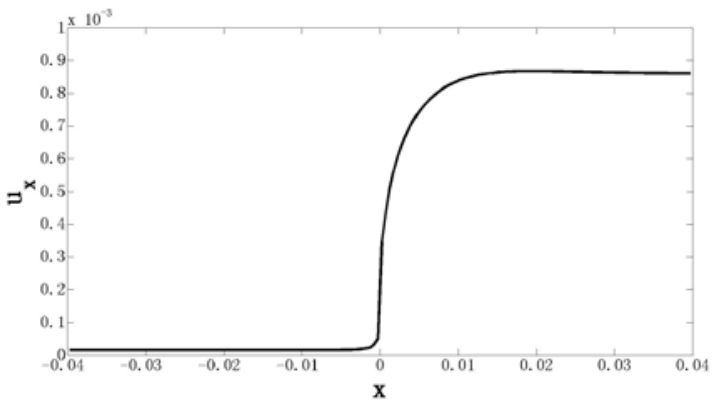

(b) $u_{x}$

Fig.3. The pressure and the $x$ component of velocity near the wall and the free surface around the singularity at $\mathrm{Re}=1$

Increasing the Reynolds number by augmenting the input velocity gradually, the swelling ratio for various Reynolds number is illustrated in Fig 4. It can be seen that the swelling ratio would decrease gradually with the increase of Reynolds number. Moreover, the swelling ratio is approaching 1 and 0.92 when the Reynolds number equals to 8 and 20 respectively, which is very close to the results in [4]. These results again demonstrate the correctness and effectiveness of our numerical algorithm.

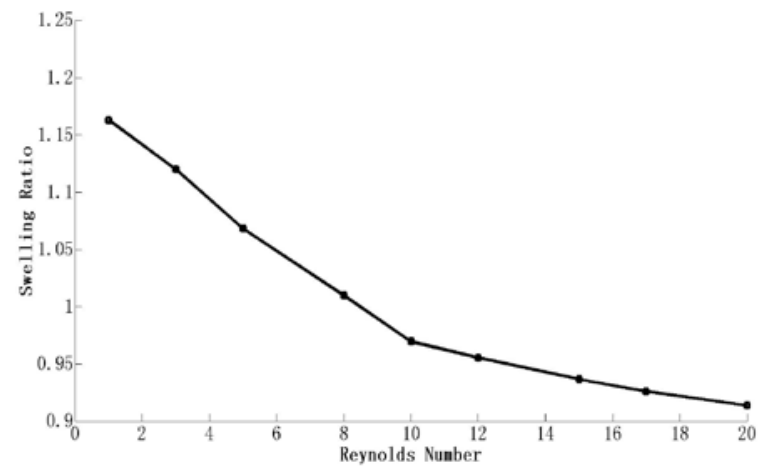

Fig.4. Swelling ratio with different Reynolds number

\section{Conclusion}

This paper proposed a new method to combine ALE with FVM. The pressure boundary condition on the surface was derived from the original dynamic boundary condition, while the according velocity boundary condition was obtained by a simple iteration process. The method was verified by extrusion flows with Newtonian fluids. The results shown that our new algorithm plays a better performance in the simulation of extrusion flows. Moreover, this method could obtain a comparable result with FEM, which again indicates the correctness and the effectiveness of this methods. More free surface simulations could be implemented with this method in the future.

\section{Acknowledgement}

Thank to the fund from the National Natural Science Foundation of China (No.61221491, No.61303071, and No.61303068), and Open fund (No.201303-01, No.201303-02) from State Key Laboratory of High Performance Computing.

\section{References}

[1] Crochet, M.J. and R. Keunings, Finite-Element Analysis of Die Swell of a Highly Elastic Fluid. Journal of Non-Newtonian Fluid Mechanics, 1982. 10(3-4): p. 339-356.

[2] Tome, M.F., et al., A finite difference technique for simulating unsteady viscoelastic free surface flows. Journal of Non-Newtonian Fluid Mechanics, 2002. 106(2-3): p. 61-106. 
[3] Russo, G. and T.N. Phillips, Spectral element predictions of die-swell for Oldroyd-B fluids. Computers \& Fluids, 2011. 43(1): p. 107-118.

[4] Mitsoulis, E., G.C. Georgiou, and Z. Kountouriotis, A study of various factors affecting Newtonian extrudate swell. Computers \& Fluids, 2012. 57: p. 195-207.

[5] Georgiou, G.C. and A.G. Boudouvis, Converged solutions of the Newtonian extrudate-swell problem. International Journal for Numerical Methods in Fluids, 1999. 29(3): p. 363-371.

[6] Favero, J.L., et al., Viscoelastic fluid analysis in internal and in free surface flows using the software OpenFOAM. Computers \& Chemical Engineering, 2010. 34(12): p. 1984-1993.

[7] Osher, S. and J.A. Sethian, Fronts Propagating with Curvature-Dependent Speed - Algorithms Based on Hamilton-Jacobi Formulations. Journal of Computational Physics, 1988. 79(1): p. 12-49.

[8] Mckee, S., et al., The MAC method. Computers \& Fluids, 2008. 37(8): p. 907-930.

[9] Hirt, C.W., A.A. Amsden, and J.L. Cook, An arbitrary Lagrangian-Eulerian computing method for all flow speeds (Reprinted from the Journal of Computational Physics, vol 14, pg 227-253, 1974). Journal of Computational Physics, 1997. 135(2): p. 203-216.

[10] Tukovic, Z. and H. Jasak, A moving mesh finite volume interface tracking method for surface tension dominated interfacial fluid flow. Computers \& Fluids, 2012. 55: p. 70-84.

[11] Jasak, H., OpenFOAM: Open source CFD in research and industry. International Journal of Naval Architecture and Ocean Engineering, 2009. 1(2): p. 89-94.

[12]Guo, X.-W., et al., Interface instabilities and chaotic rheological responses in binary polymer mixtures under shear flow. RSC Advances, 2014. 4(105): p. 61167-61177.

[13] Guo, X.-W., et al., Non-equilibrium steady states of entangled polymer mixtures under shear flow. Advances in Mechanical Engineering, 2015. 7(6): p. 1-10. 